\title{
Intervenção com atividade física em uma Unidade Básica de Saúde - Projeto UBS+Ativa: Aspectos metodológicos
}

\author{
Intervention with physical activity in one Basic Health Unity - Ubs+Ativa \\ Project: methodological aspects \\ Leony M Galliano'; Thamires L Seus',2; Márcio B Peixoto; William Macedo da Silva'; Denise S da Silveira4; Fabrício Boscolo \\ Del Vecchio'; Pedro C Hallal'; Fernando V Siqueira
}

\begin{abstract}
Resumo
O Sistema Único de Saúde é o principal meio de acesso à saúde em nível populacional. A prática atividade física regular é reconhecidamente comportamento capaz de prevenir doenças crônicas e interage de forma positiva com outras estratégias comportamentais de promoção da saúde. O objetivo deste estudo é descrever a metodologia do Projeto UBS+Ativa, programa de intervenção com atividade física em Unidade Básica de Saúde (UBS) localizada em Pelotas/RS. A intervenção é baseada em quatro atividades específicas: a) Estudo de linha de base; b) Atividade de divulgação; c) Grupo de atividade física orientada; d) Atendimento ambulatorial. Na linha de base, foram entrevistadas 601 residentes da área de abrangência da UBS. A divulgação do projeto visava recrutar participantes para o grupo de atividade física e para o atendimento ambulatorial individualizado; >2.500 folhetos foram distribuídos. Também, 470 pacientes foram abordados na sala de espera e receberam uma explicação presencial sobre o projeto e a importância da atividade física para a saúde. As aulas coletivas e o atendimento ambulatorial ocorrem três vezes por semana. Tal intervenção tem o potencial para ser ampliados localmente e nacionalmente.
\end{abstract}

\section{Palavras-chave}

Atividade motora; Atenção básica à saúde; Sistema Único de Saúde; Estudos de intervenção

\begin{abstract}
In Brazil, the Unified Health System is the main provider of health care to the population. Practice regular physical activity behavior is admittedly able to prevent chronic diseases and interacts positively with other behavioral health promotion strategies. The aim of this study is to describe the methodological aspects of a physical activity intervention - "UBS+ Ativa", taking place in primary bealth care in Pelotas, a Southern Brazilian city. The intervention was organized into four specific activities: (a) baseline survey; (b) divulgation strategies; (c) group physical activity classes; (d) individual physical activity prescription. In the baseline study, 601 individuals living in the catchment area of the primary health care unit were interviewed. The divulgation of the project was aimed at recruiting participants for the group physical activity classes and for the individual prescription appointments; >2,500 leaflets were distributed. Also, 470 patients were approached in the waiting room and obtained a face-to-face explanation about the project and the importance of physical activity for health. Group classes and individual consultations take place three times per week. This intervention has the potential to be scaled up locally and then nationally.

Keywords

Motor Activity; Primary Health Care; Unified Health System; Intervention Studies.
\end{abstract}

\section{Introdução}

O Sistema Único de Saúde (SUS) brasileiro foi criado

1 Programa de Pós-Graduação em Educação Física - Universidade Federal de Pelotas ESEF/UFPel, Pelotas, RS, Brasil.

2 Universidade Católica de Pelotas/UCPel, Pelotas, RS, Brasil.

3 Programa de Pós-Graducação em Epidemiologia - Universidade Federal de Pelotas PPGE/UFPel, Pelotas, RS, Brasil.

4 Faculdade de Medicina - Departamento de Medicina Social - Universidade Federal de Pelotas - UFPel, Pelotas, RS, Brasil. pela Constituição de 1988 e suas principais características são universalidade, promoção da equidade e da integralidade da atenção ${ }^{1}$. Suas ações estão orientadas a partir da Atenção Básica à Saúde (ABS), que objetiva ser o primeiro contato da população para acessar os serviços de saúde, além de oferecer cuidado contínuo aos usuários ${ }^{2}$. Dentro do modo de operação da ABS se localizam as Unidades Básicas de Saúde 
(UBS), que são locais destinados à promoção da saúde, a prevenção de doenças, ao diagnóstico, ao tratamento e a reabilitação dos pacientes. São consideradas como porta de entrada para o SUS com função de oportunizar para a população o acesso às ações primárias de saúde, que preferencialmente devem possuir áreas de abrangência territorial definidas e equipes multidisciplinares ${ }^{2,3}$.

A fim de atingir os seus objetivos, o SUS tem estimulado a criação de equipes de saúde da família com foco na promoção da saúde e na tentativa de se aperfeiçoar continuamente, promove diferentes avanços, tais como a reorganização da ABS, a partir da Estratégia Saúde da Família (ESF); a implantação dos Núcleos de Apoio à Saúde da Família (NASF) e, mais recentemente, em 2011, com a criação do Programa Academia da Saúde, que visa estruturar polos em 2000 cidades do país com recursos e quadro de pessoal qualificado para a orientação de atividade física e promoção de estilos de vida saudáveis ${ }^{3,4}$. Além deste esforço nacional, as intervenções de promoção da saúde locais foram identificadas e avaliadas nos últimos anos ${ }^{5,6}$. Uma delas foi implementada em 2001 com foco na promoção da atividade física em ambientes de cuidados de saúde primários, atividades cardiorrespiratórias e musculares fortes que são realizadas duas vezes por semana durante uma hora ${ }^{7}$.

Atualmente a inatividade física é responsável por 5.3 milhões de mortes por ano em todo o mundo, particularmente relacionadas a doenças cardíacas coronarianas, diabetes e alguns tipos de cânceres ${ }^{8,9}$. Recentemente foi demonstrado que um terço dos adultos são fisicamente inativos ${ }^{8}$. Em Pelotas, cidade do Sul do Brasil, a prevalência de inatividade foi estimada em 54\% em $2012^{10}$.

Em um estudo realizado na área de abrangência de UBS nas regiões Sul e Nordeste do país constatou que $32 \%$ dos adultos e $58 \%$ dos adultos mais velhos eram fisicamente inativos ${ }^{11}$. Considerando as atividades que são realizadas em academias, estudo no Brasil mostra que apenas 1,9\% da população pratica atividade física regularmente ${ }^{12}$. Em Pelotas, $7,8 \%$ da população já se inscreveram em academias de ginástica ao menos uma vez na vida ${ }^{12}$.

Sendo as UBS acessíveis a todos os cidadãos no Brasil ${ }^{13}$, a promoção da atividade física nestes locais é essencial. Infelizmente, dados sugerem que apenas 29\% dos adultos e $39 \%$ dos idosos recebem aconselhamento sobre atividade física em alguma UBS ${ }^{14}$. Há evidências de que intervenções de atividade física em cuidados primários à saúde podem ser eficazes na melhoria da saúde e qualidade de vida. Este artigo tem o objetivo de descrever as características metodológicas de uma intervenção baseada em atividade física realizada em uma UBS e na sua área de abrangência, chamada de "UBS + Ativa".

\section{Métodos}

\section{Tipo de estudo e delineamento operacional}

O Projeto UBS+Ativa, aprovado pelo Comitê de Ética em Pesquisa da Escola de Educação Física da Universidade Federal de Pelotas, sob protocolo número 224.894/2013, é um estudo de intervenção que acontece a partir de programa de atividade física em área de abrangência de uma UBS de Pelotas, cidade do sul do Rio Grande do Sul - Brasil. Esta cidade possui área de 1.921.80 Km exibe clima subtropical, com média anual de $17,6^{\circ} \mathrm{C}$ e umidade relativa do ar bastante elevada, com média anual de $85 \%$, além de chuvas distribuídas durante todo o ano ${ }^{15}$. Demograficamente, possui aproximadamente 330 mil habitantes ${ }^{16}$.

A UBS, intencionalmente selecionada, foi definida com base nos seguintes critérios: i) estar sob gestão da Universidade Federal de Pelotas; ii) atender, de segunda a sexta-feira, nos turnos da manhã e da tarde; iii) contar com espaço interno 
suficiente para atendimento ambulatorial e externo que possibilitasse a realização de atividades coletivas supervisionadas e iv) possuir cobertura de Agente Comunitário de Saúde. A população da área de abrangência se caracteriza por maioria de mulheres, cor de pele branca, que vive com companheiro, sabe ler e escrever e com três pessoas morando por domicílio.

\section{Procedimentos de coleta e registro dos dados populacionais}

Fez parte desta estratégia duas avaliações na população da área de abrangência da UBS, o Estudo de Linha de Base (ELB - A1) e a Avaliação Final (A2). Afim de melhor operacionalizar a coleta de dados, a área de abrangência da UBS foi dividida em sete micro-áreas com aproximadamente 400 domicílios em cada uma. Em cada uma das micro-áreas, um domicílio sorteado representou o ponto inicial da coleta de dados. Todos os moradores da residência sorteada com idade a partir dos 18 anos foram entrevistados. Em sequência, o entrevistador posicionado de costas para a porta de entrada deslocava-se à direita, considerando um pulo de cinco domicílios e no seguinte, ou seja, no sexto domicílio, aplicava o instrumento em seus residentes na faixa etária definida. Este procedimento finalizava quando todas as ruas da tivessem sido abrangidas.

Após doze meses do primeiro inquérito, e, portanto, da intervenção, os mesmos indivíduos entrevistados no primeiro momento foram novamente visitados para nova avaliação (A2). O intuito desta ação foi verificar o impacto de intervenção com atividade física na população da área de abrangência da UBS.

Para caracterização da amostra, as seguintes variáveis demográficas foram consideradas: sexo (masculino/feminino), idade (anos completos), cor da pele (branca/parda/preta), saber ler e escrever (não/sim/só assina o nome), escolaridade (não estudou/ fundamental/médio/superior), situação conjugal (com companheiro[a]/ sozinho[a]) e quantas pessoas residiam no domicílio (uma/duas/três/quatro/cinco ou mais).

\section{Desfechos do estudo}

Os desfechos primários do estudo foram o nível de atividade física da população, registrado a partir do IPAQ ${ }^{17}$, considerando sessões lazer e deslocamento e o recebimento de orientação médica para prática de atividade física, operacionalizado pela pergunta: "Alguma vez, em uma consulta na UBS, algum médico lhe disse que o(a) Sr.(a) deveria fazer atividade física para melhorar a sua saúde?”14.

Entre os desfechos secundários foram considerados: consulta na UBS Areal -Leste ( $\operatorname{sim} /$ não), índice de massa corporal (IMC, tendo como valores de referência - baixo peso: $<18.5 \mathrm{~kg} / \mathrm{m}^{2}$; normal: $18.6-24.9 \mathrm{~kg} / \mathrm{m}^{2}$; sobrepeso: $25-29.9 \mathrm{~kg} / \mathrm{m}^{2} \mathrm{e}$ obeso: $\left.\geq 30 \mathrm{~kg} / \mathrm{m}^{2}\right)^{18}$, circunferência da cintura $(\mathrm{em} \mathrm{cm})^{19}$, pressão arterial sistólica e diastólica (em $\mathrm{mmHg})^{20}$, percepção de saúde (excelente, muito boa, boa, regular, ruim), queda no último ano ( $\mathrm{sim} /$ não), aconselhamento médico para perder peso ( $\operatorname{sim} /$ não), autorrelato de hipertensão arterial sistêmica ( $\operatorname{sim} /$ não) e de diabetes mellitus (sim/não).

A massa corporal foi obtida através do uso de balança digital (Geratherm ${ }^{\circledR}$ ) sendo o entrevistado orientado a permanecer descalço, com o mínimo de roupa possível, mantendo-se ereto e pisando no centro da balança. Para verificação da estatura, o avaliado permanecia em pé, olhando para frente, com os pés juntos e voltados para frente, ombros relaxados e braços ao longo do corpo. A aferição da circunferência da cintura foi obtida na região mais estreita do tronco entre as costelas e a crista ilíaca (risco elevado $>94 \mathrm{~cm}$ (homens) e $>80 \mathrm{~cm}$ (mulheres)) ${ }^{21}$. Para ambas as medidas foram utilizadas trenas WISO ${ }^{\circledR}$, Modelo T87. A pressão arterial 
foi aferida com aparelho de pressão Geratherm Wristwatch Medical $A G^{\mathrm{TM}}$. Neste procedimento, o indivíduo estava sentado com a coluna ereta e punho na altura do coração. A braçadeira foi ser posicionada no pulso de modo a ficar do mesmo lado da palma da mão e, então, mensurada por oscilometria ${ }^{22 .}$ Os resultados obtidos foram anotados em fichas individuais.

\section{Controle de qualidade, processamento e análise dos dados}

O controle de qualidade na coleta de dados A1 e A2 da intervenção, foram realizados pelo supervisor de cada uma das micro-áreas de estudo. Os entrevistadores eram acompanhados diariamente pelo supervisor com o objetivo de verificar a seleção correta dos domicílios de acordo com a metodologia proposta, a verificação da aplicação do instrumento a todos os moradores elegíveis do domicílio e a verificação do preenchimento correto do instrumento.

O processamento dos dados incluiu o recebimento do instrumento, a sua identificação e a codificação das perguntas fechadas. A estrutura da base de dado foi criada no programa EpiData (http://www.epidata.dk./), incluindo a verificação da amplitude e consistência das variáveis. Após esta etapa, todos os instrumentos passaram por dupla digitação. Toda a base de dados foi exportada para diferentes pacotes estatísticos que possibilitaram as análises de dados. Os dados coletados permitem estratificação de acordo com a existência ou não de atendimento na área por agentes comunitários de saúde e distância da moradia até a UBS.

\section{Protocolo da intervenção}

A intervenção do Projeto UBS+Ativa foi composta de diferentes níveis, a saber:

\section{- Nível 1 - Divulgação}

A principal finalidade desta frente de intervenção foi divulgar: i) o projeto, ii) a inserção do profissional de Educação Física na UBS que recebeu a intervenção e iii) a relevância da atividade física e do estilo de vida ativo para desfechos em saúde. Neste sentido, foram executadas duas estratégias para atingir este objetivo e envolver os moradores da região na participação do projeto, organizadas em dois grandes grupos: divulgação passiva e ativa.

Considerou-se como divulgação passiva: a) a exposição de cartazes e faixas com informações básicas sobre as aulas coletivas e atendimento ambulatorial; b) informações e esclarecimentos sobre a prática de atividade física (dia das atividades e horários) em veículos midiáticos fixados em áreas de grande circulação da população alvo (ex.: na parte interna e externa da UBS, transporte público da região de abrangência da UBS); c) vídeo institucional com informações sobre os benefícios da atividade física, hábitos de vida saudável para prevenir doenças e promoção da saúde e qualidade de vida veiculado na sala de espera da UBS e, d) banner para comunicação visual na sala de espera.

A divulgação ativa foi realizada considerando-se ações de panfletagem na área de abrangência da UBS após o quarto mês da intervenção, com entrega de folders diretamente aos moradores, e quando isso não fosse possível, o mesmo era deixado na caixa de correspondência das residências. Este material continha informações semelhantes as dos cartazes, pôsteres e vídeo. Além disso, durante esta ação, os moradores abordados eram convidados a participar do projeto, tanto nas práticas coletivas, quanto do atendimento ambulatorial.

Simultaneamente, era realizada na UBS uma conversa com "roteiro dialógico", previamente preparado, no qual diariamente um membro do projeto previamente 
treinado, era o responsável por conduzir o diálogo com os usuários que estavam na sala de espera da UBS aguardando atendimento. Nesta oportunidade, era desenvolvida uma fala previamente padronizada sobre o projeto e suas principais ações, assim como, explanação acerca dos benefícios da atividade física, com informações básicas sobre alimentação, consumo de álcool e tabagismo ${ }^{23,24}$. No final desta conversa, de acordo com a avaliação realizada e o interesse do paciente, este era aconselhado às atividades de aulas coletivas e/ou atendimento ambulatorial. Para esta ação, além do roteiro dialógico semi-estruturado, os interventores portavam instrumentos educativos impressos que orientavam e ilustravam as falas e exemplos.

\section{- Nível 2 - Práticas coletivas:}

O Grupo de Atividade Física Orientada (GAFO) foi uma das estratégias do projeto que proporcionava oportunidade para a prática de exercícios físicos coletivamente. Entre as atividades, o grupo de caminhada representava um método de baixa complexidade, de fácil execução e benéfico para o sistema cardiovascular ${ }^{25}$. As práticas eram lideradas com supervisão profissional por graduandos em Educação Física e aconteciam em dois dias da semana nos períodos da manhã e da tarde, em pista de caminhada próxima à UBS.

Além da atividade de caminhada, sessões de exercícios eram realizados semanalmente, com duração média entre 60-75 minutos com intensidade baseada na Escala de Percepção Subjetiva de Esforço de Borg aplicada para exercício resistido $^{25,26}$. A progressão da intensidade ocorria $\operatorname{com}^{27}$ : a) aumento da proporção esforço-repouso; b) diminuição do tempo de descanso entre as séries e aumento do tempo de atividade na parte principal. Cada sessão era composta por atividades aeróbias, treino de resistência e força muscular. No período inicial (5-10 min) aquecimento e alongamentos musculares, na parte principal realização de esforços em circuito ou na forma intervalada com exercícios aeróbios e de resistência (30-35 min) composto por exercícios multi-articulares (remada curva, agachamento, supino reto, remada alta e rosca bíceps com extensão) e 10-15 min de flexibilidade. No final, eram executados alongamentos e relaxamentos por aproximadamente $10 \mathrm{~min}$.

\section{- Nível 3 - Atendimento ambulatorial:}

Os usuários com diferentes morbidades e que não apresentavam condições de saúde ou disponibilidade de tempo para se engajarem nas aulas coletivas eram encaminhados ao atendimento ambulatorial individualizado. Neste momento, o profissional de Educação Física conversava com o usuário ${ }^{23}$, realizava a avaliação inicial (composta por entrevista sobre histórico de doenças, uso de medicamentos, dores no corpo, tabagismo, aplicação do instrumento para avaliação da qualidade de vida SF-8, questionário sobre atividades físicas IPAQ - domínio do lazer, e hábitos relacionados à atividade física) e prescrevia atividade física, indicando o tipo, frequência e duração dos exercícios físicos ${ }^{28,29}$ bem como, locais apropriados para a prática e presença de outros programas comunitários nos quais este poderia se engajar, considerando as necessidades e possibilidades individuais.

Os demais profissionais da UBS, como médicos, enfermeiros e nutricionistas, foram estimulados a referenciarem para o atendimento os pacientes que, segundo os seus protocolos profissionais de atendimento, necessitavam de avaliação e prescrição de atividade física. Neste caso era realizada, além da conversa com os demais profissionais sobre o paciente, a leitura e a revisão do prontuário para direcionamento do atendimento que ocorreriam em dias específicos da semana, conforme a 
história pregressa do usuário. Como estratégia de distribuição dos atendimentos, agravos cardiometabólicos eram atendidos nas segundas-feiras, transtornos psíquicos menores nas quartas-feiras e problemas osteomusculares às sextas-feiras.

Uma semana após o atendimento ambulatorial, o usuário era procurado por ligação telefônica para execução de entrevista semi-estruturada sobre o desenvolvimento prático do programa de exercícios indicado e para que fossem sanadas dúvidas em relação à prescrição, caso ainda existissem. Nesta ligação é perguntado: a) o $\mathrm{Sr}(\mathrm{a})$ está realizando as atividades prescritas? Caso a resposta fosse negativa, pergunta-se o motivo para a não realização da prescrição indicada; b) O Sr(a) permanece com alguma dúvida em relação a prescrição que está dificultando a realização deste plano? Caso existisse alguma dúvida a estratégia do projeto foi de procurar sanar a mesma durante a ligação ou agendar nova consulta para o indivíduo.

Após duas semanas o paciente recebia visita de agente comunitário de saúde para identificação de pontos relevantes e esclarecimento sobre possíveis dúvidas.

Um mês após o primeiro atendimento, era realizado agendamento para o segundo atendimento. Todas as prescrições realizadas para os indivíduos, nos atendimentos ambulatoriais, eram disponibilizadas para toda a equipe da UBS, em prontuário do paciente para que pudessem ser consultadas.

\section{Fluxograma e Logística do Projeto de Intervenção}

As etapas do projeto e suas respectivas ações estão apresentadas na Figura 1. Nela, observam-se os diferentes níveis de complexidade, suas frentes de ação e desdobramentos.

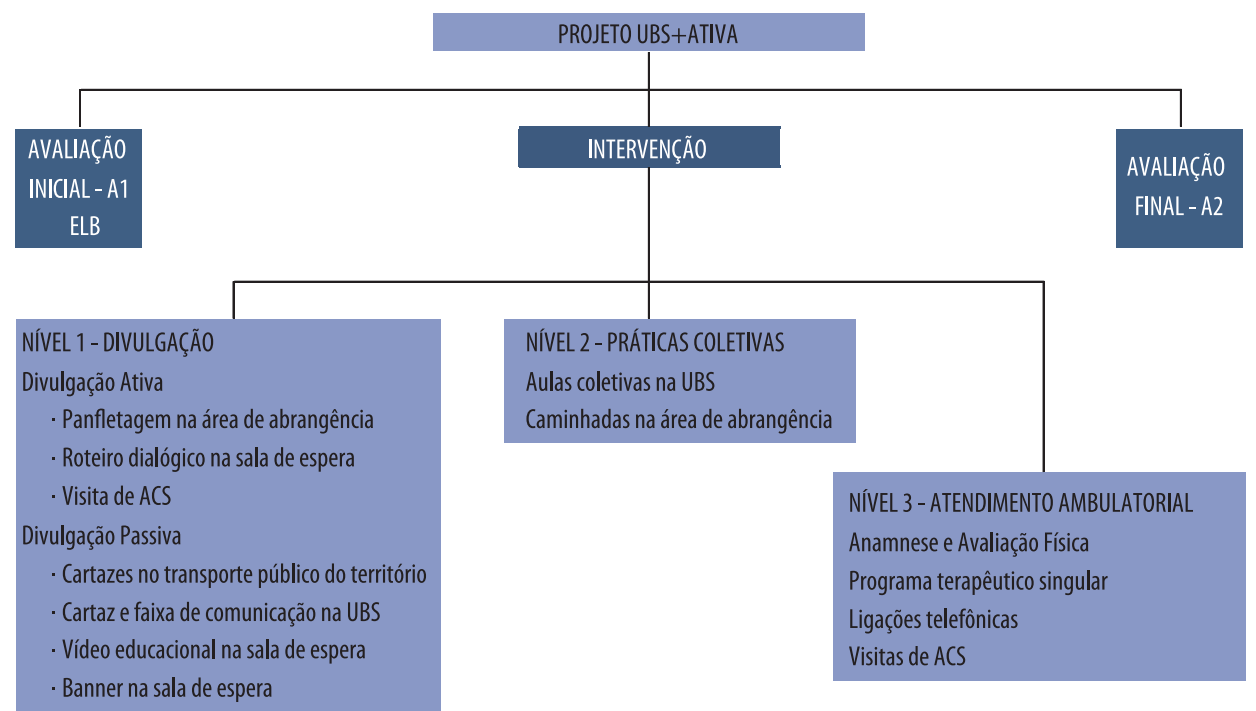

UBS = Unidade básica de saúde. ACS = Agente comunitário de saúde. ELB = Estudo de linha de base. FIGURA 1 - Fluxograma do projeto de intervenção "UBS+Ativa” (ESEF, UFPel, 2013).

\section{Estudo populacional comunitário na área de abrangência da UBS}

Depois de tecidas as relações iniciais com a equipe multiprofissional da UBS para apresentação da intervenção, a primeira ação desenvolvida foi a realização da Avaliação Inicial - Estudo de Linha de Base Populacional (ELB - A1) com o intuito de conhecer e registrar as características dos adultos de 18 anos ou mais e dos idosos residentes na área de abrangência da UBS, no que tange às variáveis demográficas, de saúde, busca de atendimento em saúde e questões relacionadas à prática de atividade física regular. Esta avaliação foi reconduzida 12 meses após a aplicação das ações de intervenção (Avaliação Final - A2). 


\section{População Estudada e Amostra}

A amostra do estudo foi composta pela população residente na região de cobertura da UBS que soma aproximadamente 10 mil moradores. Estimou-se que neste estudo seriam entrevistados, em domicílio, cerca 870 indivíduos de 18 anos ou mais. Considerando previsão de concentração demográfica de 1,94 habitantes adultos por domicilio e de 0,32 idosos ${ }^{30}$, estimou-se visitar 55 domicílios em cada uma das sete micro-áreas da área de abrangência.

\section{Resultados}

\section{Estudo de Linha de Base e Reavaliação}

A implementação da metodologia do estudo exigiu desafio logístico em decorrência da realidade da área de abrangência, que apresentava necessidades em termos de estrutura viária, saneamento e segurança. No entanto, o percentual de completude da coleta de dados, a possibilidade de execução em termos da logística para a realização do trabalho de campo, mostrou-se factível e reprodutível para as mais diferentes áreas de UBS. Assim, o trabalho de da linha de base foi realizados em 14 dias no mês de março de 2013, enquanto a reavaliação da intervenção durou 30 dias, entre abril e maio de 2014, ambas contemplando as sete micro-áreas referentes a área de abrangência da UBS. Na linha de base foram obtidos dados de 713 pessoas elegíveis (7,13\% da população da área da UBS), sendo que 601 foram entrevistadas (84,3\%), 70 foram perdas $(9,8 \%)$ e 42 recusas $(5,9 \%)$, num total de perdas e recusas de $15,7 \%$.

Estratégias de divulgação ativa e passiva foram realizadas na área de abrangência da UBS. A panfletagem, estratégia de divulgação ativa, ocorreu durante um dia após o quarto mês de intervenção, em agosto de 2013, e envolveu os entrevistadores e supervisores do estudo com distribuição de 2.500 folders promocionais do projeto na área de abrangência. Para esta estratégia, foram consideradas todas as ruas da área total de abrangência da UBS. Para garantir uma maior distribuição em relação à área adstrita, foi considerando o número total de folders disponíveis $(\mathrm{n}=2.500)$ divididos pelo número aproximado de 2.800 domicílios, resultando em um pulo de um domicílio para orientar o andar pelo território. Ao final, 89,2\% $(\mathrm{n}=2.497)$ dos domicílios receberam a visita para distribuição do folder. Estima-se que com esta logística e considerando a cota de 3,3 habitantes por domicílio, que a estratégia tenha atingido 8.242 indivíduos moradores da área. A atividade iniciou às $9 \mathrm{~h}$ da manhã e ao meio da tarde estava terminada.

Em relação às ações na sala de espera, semanalmente os usuários da UBS eram abordados por indivíduos do projeto para falar sobre a intervenção e atividade física. Ao total, foram abordados 470 indivíduos nesta ação, 80,2\% do sexo feminino. Dois banners informativos foram confeccionados e colocados na UBS em local visível, contendo informações sobre a importância da prática de atividade física e da manutenção de estilo de vida saudável e os horários das atividades. Faixa promocional foi colocada na área externa da UBS e serviu como meio de divulgação das ações. Um vídeo institucional de divulgação do projeto foi editado e disponibilizado por meio eletrônico na página http:youtu.be/DXOrzAiZ86Y e, foi criada uma página eletrônica de divulgação das ações do grupo em www.geeafab.com.

As atividades coletivas foram implementadas conforme previsto na metodologia da intervenção e aconteciam em três turnos por semana. Durante os doze meses verificou-se que os dias nublados e os meses relacionados com a estação de outono foram aqueles com maior frequência de alunos. Para a realização destas atividades foram utilizados espaços para caminhada na área contígua a UBS, as- 
sim como o pátio da UBS e uma área próxima disponibilizada por uma associação da comunidade. Tais estratégias resultaram em excelentes possibilidades a serem utilizadas na intervenção.

Em relação ao atendimento ambulatorial foi implantado na UBS em três dias semanais, com capacidade de atendimento de três pessoas por dia por profissional de educação física $(n=3)$, atingindo, portanto nove atendimentos/semana, 36 por mês e 432 ao ano.

\section{Considerações finais}

A proposta de intervenção apresentada permitiu que se somassem aos profissionais que tradicionalmente compõe a equipe de atendimento da UBS, os profissionais de educação física, possibilitando a realização de diversas atividades relacionadas à promoção, prevenção e atendimento a saúde que até o início da intervenção não era realizada por profissional específico na UBS. Além disso, a presença deste profissional permitiu aumentar o leque de atendimento as necessidades dos usuários e trabalhar pelo aumento da resolutividade na própria UBS. Ainda, acrescentou diversas ações positivas que não eram realizadas tais como, as intervenções na sala de espera, os grupos de atividade física orientada e o atendimento ambulatorial, importantes ferramentas para apoiar as ações dos médicos, enfermeiros e demais profissionais da UBS.

Salienta-se também, como importante o fato da população estar coberta pelo Programa de Agentes Comunitários de Saúde, e este programa auxiliar positivamente na divulgação da intervenção pela proximidade e característica do trabalho deste grupo de profissional da saúde na área de abrangência da UBS.

Importante também, que a implementação da intervenção por níveis de complexidade, possibilitou maior clareza das etapas a serem implementadas para todos os profissionais que atuam na UBS, o que determinou e facilitou o comprometimento de toda a equipe com as ações previstas.

Consideramos que através deste estudo será possível avaliar o impacto de um programa de intervenção à população de área adstrita de UBS ao longo do tempo, em relação aos indicadores de saúde na comunidade, aos esforços empregados no programa de intervenção e sua relevância no âmbito da saúde. Da mesma forma, considerando que o estudo foi desenvolvido a partir de amostra comunitária, ou seja, composta por usuários e não usuários da UBS, será possível avaliar o desempenho do Projeto UBS+Ativa para algumas variáveis, como o aconselhamento para a prática de atividade física, a mudança de comportamento em relação ao estilo de vida, o aumento do conhecimento sobre os benefícios da atividade física e o impacto causado na população em relação aos níveis de atividade física no deslocamento e no lazer e, em relação a alguns desfechos em saúde tais como hipertensão e diabetes.

Considerando a metodologia proposta e aplicada no projeto "UBS+ATIVA", bem como os resultados apresentados até o momento em relação a sua implementação metodológica, destaca-se a possibilidade da aplicação desta metodologia para projetos de intervenção em outras UBS do município.

\section{Contribuiç̧ões dos autores}

LMG participou de todas as fases do artigo, desde sua concepção, coleta de dados até a escrita. TLS, MBP, WMS participaram da coleta de dados e escrita do artigo. DSS participou da escrita do manuscrito. FBDV, PCH, FVS supervisionaram todas as etapas do trabalho. Todos os autores participaram da escrita do artigo, 
dando contribuições intelectuais para o aprimoramento do mesmo. Todos os autores aprovaram a versão submetida para publicação.

\section{Agradecimentos}

\section{FAPERGS}

\section{Referências}

1. Brasil. Ministério da Saúde. Secretaria de Políticas de Saúde. Projeto Promoção da Saúde. As Cartas da Promoção da Saúde / Ministério da Saúde, Secretaria de Políticas de Saúde, Projeto Promoção da Saúde. - Brasília: Ministério da Saúde, 2002.

2. Brasil. Ministério da Saúde. Secretaria de Vigilância em Saúde. Política nacional de promoção da saúde / Ministério da Saúde, Secretaria de Atenção à Saúde. - Brasília: Ministério da Saúde, 2006.

3. Portaria $n^{\circ} 2.488$, de 21 de outubro de 2011. Aprova a Política Nacional de Atenção Básica, estabelecendo a revisão de diretrizes e normas para a organização da Atenção Básica, para a Estratégia Saúde da Família (ESF) e o Programa de Agentes Comunitários de Saúde (PACS). Diário Oficial [da República Federativa do Brasil], Brasília 2011; 204:55.Seção 1, pt1.

4. Brasil. Ministério da Saúde. Portaria GM n.154, de 24 de janeiro de 2008. Cria os núcleos de Apoio à Saúde da Família - NASF. Brasília; 2008. Diário Oficial da União 25 Jan 2008. [n. 18].

5. Reis RS, Hallal PC, Parra DC, Ribeiro IC, Brownson RC, Pratt M, et al. Promoting physical activity through community-wide policies and planning: findings from Curitiba, Brazil. J Phys Act Health, 2010; 2(7):137-45

6. Nakamura PM, Papini CB, Teixeira IP, Chiyoda A, Luciano E, Cordeira KL, et al. Effect of a 10-Year Physical Activity Intervention in Primary Health Care Settings on Physical Fitness. J Phys Act Health. 2014; 12(1):102-8.

7. Kokubun E, Luciano E, Sibuya CY, Queiroga MR, Ribeiro PAB, Silveira RF, et al. Programa de atividade física em unidades básicas de saúde: relato de experiência no município de Rio Claro-SP. Rev Bras Ativ Fis e Saúde. 2007; 1(2):45-50.

8. Hallal PC, Andersen LB, Bull FC, Guthold R, Haskell W, Ulf Ekelund. Global physical activity levels: surveillance progress, pitfalls, and prospects. The Lancet. 2012; 380(9838):247-57.

9. Lee IM, Shiroma EJ, Lobelo F, Puska P, Blair SN, Katzmarzyk PT. Effect of physical inactivity on major non-communicable diseases worldwide: an analysis of burden of disease and life expectancy. The Lancet. 2012; 380(9838):219-29.

10. Hallal PC, Cordeira K, Knuth AG, Mielke GI, Victora CG. Ten-year trends in total physical activity practice in Brazilian adults: 2002-2012. J Phys Act Health. 2014; 11(8):1525-30.

11. Siqueira FV, Facchini LA, Piccini RX, Tomasi E, Thumé E, Silveira DS, et al. Atividade física em adultos e idosos residentes em áreas de abrangência de unidades básicas de saúde de municípios das regiões Sul e Nordeste do Brasil. Cad. Saúde Pública. 2008; 24(1):39-54.

12. Silva MC, Rombaldi AJ, Azevedo MR, Hallal PC. Participação atual e passada em academias de ginástica entre adultos: prevalência e fatores associados. Rev Bras Ativ Fis e Saúde. 2008; 13(1):28-36.

13. Paim J, Travassos C, Almeida C, Bahia L, Macinko J. The Brazilian health system: history, advances, and challenges. The Lancet. 2011; 377(9779):1778-97.

14. Siqueira FV, Nahas MV, Facchini LA, Silveira DS, Piccini RX, Tomasi E, et al. Aconselhamento para a prática de atividade física como estratégia de educação à saúde. Cad. Saúde Pública. 2009; 26(1):203-13.

15. Universidade Federal de Pelotas (UFPEL). Pelotas - Patrimônio Cultural do RS. 2013. [Acesso em: 19 de janeiro de 2014]. Disponível em: http://www2.ufpel.edu.br/pelotas/ geografia.html

16. IBGE, Censo Demográfico 2010. [Acesso 14 de novembro de 2013]. Disponível em: http://www.censo2010.ibge.gov.br/sinopse/webservice/default. php?cod $1=43 \& \operatorname{cod} 2=431440 \& \operatorname{cod} 3=43 \&$ frm $=$ evo_pop

17. Pardini R, Matsudo S, Araújo T, Matsudo V, Andrade E, Braggion G, et al. Validação do questionário internacional de nível de atividade física (IPAQ - versão 6): estudo piloto em adultos jovens brasileiros. Rev Bras Ciên Mov. 2001; 9(3):45-51. 
18. WHO. Obesity: preventing and managing the global epidemic. Report of a WHO Consultation. WHO Technical Report Series 894. Geneva: World Health Organization, 2000.

19. National Health and Nutrition Examination Survey (NHANES). Anthropometry Procedures Manual. Centers for Disease Control and Prevention. 2007.

20. National Health and Nutrition Examination Survey (NHANES). Health Tech/Blood Pressure Procedures Manual. Centers for Disease Control and Prevention. 2009.

21. WHO. Waist Circumference and Waist-Hip Ratio: Report of a WHO Expert Consultation Geneva, 8-11 December 2008.

22. Belghazi J, Feghali RNE, Moussalem T, Rejdych M, Asmar RG. Validation of four automatic devices for self-measurement of blood pressure according to the International Protocol of the European Society of Hypertension. Vasc Health Risk Manag. 2007; $3(4): 1-12$.

23. Orrow G, Kinmonth A, Sanderson S, Sutton S. Effectiveness of physical activity promotion based in primary care: systematic review and meta-analysis of randomised controlled trials. BMJ. 2012; 344:e1389.

24. Division of Nutrition, Physical Activity and Obesity, National Center for Chronic Disease Prevention and Health Promotion. Centers for Disease Control and Prevention (CDC). Physical Activity and Health The Benefits of Physical Activity. Fevereiro, 2011. [Acesso em: 07, janeiro 2014]. Disponível em: http://www.cdc.gov/physicalactivity/ everyone/health/

25. Row BS, Knutzen KM, Skogsberg NJ. Regulating explosive resistance training intensity using the rating of perceived exertion. J Strength Cond Res. 2012; 26(3):664-71.

26. Chtara M, Chaouachi A, Levin GT, Chaouachi M, Chamari K, Amri M, et al. Effect of concurrent endurance and circuit resistance-training sequence on muscular strength and power development. J Strength Cond Res. 2008; 22(4):1037-45.

27. Barcellos FC, Santos IS, Mielke GI, Del Vecchio FB, Hallal PC. Effects of exercise on kidney funcntion among non-diabetic patients with hypertension and renal disease: randomized controlled trial. BMC Nephrology. 2012; 13:90.

28. Garber CE, Blissmer B, Deschenes MR, Franklin BA, Lamonte MJ, Lee I-M, et al. Quantity and Quality of Exercise for Developing and Maintaining Cardiorespiratory, Musculoskeletal, and Neuromotor Fitness in Apparently Healthy Adults: Guidance for Prescribing Exercise. Med Sci Sports Exerc. 2011; 43(7):1334-59.

29. Chodzko-Zajko WJ, Proctor DN, Singh MAF, Minson CT, Nigg CR, Salem GJ, et al. Exercise and Physical Activity for Older Adults. Med Sci Sports Exerc. 2009; 41(7):1510-30.

30. IBGE. Censo Demográfico 2000, Instituto Brasileiro de Geografia e Estatística, 2001.

ENDEREÇO PARA

CORRESPONDÊNCIA

LEONY MORGANA GALLIANO

Imgalliano@gmail.com
Luís de Camões, 625

Bairro Três Vendas

96055-630

Pelotas, RS
RECEBIDO 20/07/2015

REVISADO 02/10/2016

APROVADO $\quad 03 / 10 / 2015$ 